\title{
Conveying Availability and Capability to Communicate in Naturalistic Interaction
}

\author{
Victoria Land \\ DWRC, University of Surrey \\ 125 Sirius House Adastral Park \\ Ipswich, Suffolk, IP5 3RE \\ +44 (0)1473643534 \\ victoria.land@bt.com
}

\author{
Mary Lumkin \\ British Telecommunications plc \\ 125 Sirius House Adastral Park \\ Ipswich, Suffolk, IP5 3RE \\ +44 (0)1473643810 \\ mary.lumkin@bt.com
}

\author{
David Frohlich \\ DWRC, University of Surrey \\ Guildford \\ Surrey, GU2 7XH \\ +44 (0) 1483683973 \\ d.frohlich@surrey.ac.uk
}

\begin{abstract}
This paper investigates the basis for social awareness; analysing naturalistic data to understand how people convey availability and capability to communicate in everyday interaction and how they use existing presence systems. The findings show that people in close personal relationships provide intermittent information about their activities and plans which are used to infer and negotiate future contact and communication decisions. The implications for more sophisticated cross-media communication systems are discussed.
\end{abstract}

\section{Categories and Subject Descriptors}

H.5.3 [Information Interfaces and Presentation]: Group and Organisation Interfaces - asynchronous interaction, collaborative computing, computer-supported collaborative work, evaluation/methodology, synchronous interaction, webbased interaction. H.5.2 [Information Interfaces and Presentation]: User Interfaces - natural language, usercentred design.

\section{General Terms}

Design, Human Factors.

\section{Keywords}

Presence, awareness, naturalistic data, communication, crossmedia.

\section{INTRODUCTION}

In our modern lives we use a plethora of devices and communication tools to manage our interactions - fixed line telephony, mobile phones, SMS, MMS, VoIP services, instant messaging, email, social networking sites and internet forums to name a few of the more popular methods. This, in turn, has made decisions about how and when to contact someone more pertinent. Social awareness involves achieving "an understanding of the activities of others, which provides a context for your own activity" [7]. In making communication decisions, presence or awareness incorporates two related

(C) The Author 2008.

Published by the British Computer Society concepts: availability of a potential recipient and their capability to communicate. Availability refers to whether a person is available to communicate, that is, whether or not they are involved in some activity that would preclude them also being engaged in an interaction. Capability indicates the means by which a person is contactable (e.g., by mobile phone and text but not video on a computer and so on). A contemporary example of these two levels of awareness would be the settings on an instant messaging system like MSN Messenger. The buddy list shows who is currently on-line and technically capable of receiving instant messages, while the busy status indicators show a level of availability to be interrupted.

Presence literature focuses heavily on workplace presence (e.g., [4] [10]). Although this research provides a useful starting point there are significant differences between work and non-work environments. For example, Xerox's 'Portholes' [8] were one of the earliest well-known awareness systems but such a system would be unlikely to be adopted in a non-work environment due to privacy issues. These systematic differences mean there are different considerations to be made in relation to presence in the social sphere. Therefore, "[t]he importance of this awareness goes beyond the workplace" [6] and communicating presence information has "a huge potential in domestic settings" [10]. The data in this paper is predominantly social/domestic, although because there is overlap there is some workplace interaction (which mirrors the general trend towards blurring of 'home' and 'work').

Dabbish and Kraut concluded that "[r]ecommendations for designing awareness displays are clear: provide an abstract display with pertinent information about a remote collaborator's task, while minimizing extraneous detail" [4]. However, what is considered 'pertinent' context is not necessarily so straightforward. Although we know we need context, in relation to human-computer interaction this is problematic as "often we don't know what contextual information is relevant, useful, or even how to use it" [12]. At the centre of design for improved presence services is the "need to find out what information a system would need" [5] which is quite difficult as during "daily interactions we are not used to making social judgments based on someone's location or phone alarm profile, but on gestures, gazes, looks, movement, talk and so on" [13]. Moreover, much of the research on presence systems is experimental and/or observed in a simulated environment (e.g., [4]). By contrast, the research described here uses naturalistic data to understand how people actually communicate information that allows their interlocutor to discern their availability and capability to communicate. Therefore, the research can identify what information is needed by examining what is relevant to participants themselves. 
A very recent study using content analysis has also used naturalistic data (although restricted only to mobile phone interaction) to understand disclosure of location and activity information between remote parties [2]. We build on this work by recording a wider range of interactions across media and using conversation analysis inspired approach to examine orientation to future communication.

\section{APPROACH}

The data corpus comprises recordings of the naturalistic interactions of eight pairs of people in key social relationships. All the communications between the pair (via every media they used) were recorded over a two week period.

The volunteers responded to adverts seeking pairs of people who communicated with each other more than ten times a week using four or more communication methods. The relationships between the pairs included best friends, partners, and parent and adult offspring. For ethical reasons, the data has been anonymised, which involves changing names, events, locations and other information that could lead to identification. The participants were active in the data collection process and were therefore free to choose not to record any communication they did not wish to include in the data they submitted.

The resulting data corpus comprised 499 actual or attempted instances of communication across an array of media, including: fixed line phone calls, mobile phone calls, voicemail, face-to-face interaction, SMS, instant messaging, email, handwritten notes, and social networking sites (e.g., Facebook and Bebo). The raw data were organised into cross-media transcripts in chronological order from the perspective of the sender/initiator. For the most part, this also reflected the experience of the receiver with the exception of a few instances in which participants might, for example, read an SMS that was sent after an email prior to reading that email. The data were characterised with broad quantitative analyses and also a qualitative method which was shaped by a conversation analytic approach. This means, among other things, that there was focus on actual communications (rather than simulated interactions or only reports of communications practices), attention to the content of the communications (rather than only the incidence of interactions), and an attempt to understand what participants do and not just what they say they do.

\section{HOW PARTICIPANTS CONVEY AVAILABILITY AND CAPABILITY TO COMMUNICATE}

Across the data corpus it is normative for participants to share information that gives their interlocutors awareness knowledge. This is not usually direct awareness information that would be included in a typical presence system but rather related information about their everyday activities and plans.

\subsection{Talking About Current and Future Activities}

In the following data fragments participants give information about their current activity (or lack thereof).

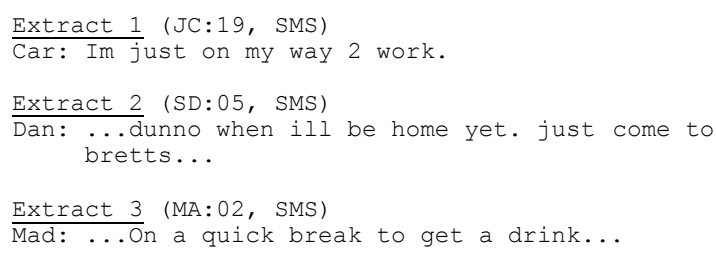

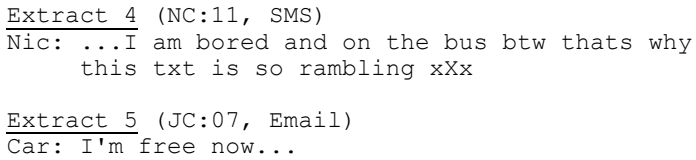

In each of these extracts the sender of the message is informing their recipient of an activity: traveling to work (Extract 1); meeting a friend at their house (Extract 2); having a quick break (Extract 3); on a bus journey (Extract 4); and doing nothing (Extract 5). Some of these also provide location information to a knowledgeable recipient, such as if the receiver of Maddy's text in Extract 3 knows what she is breaking from (and the implication is that the recipient does know).

In the next data excerpts participants provide information about their future activities.

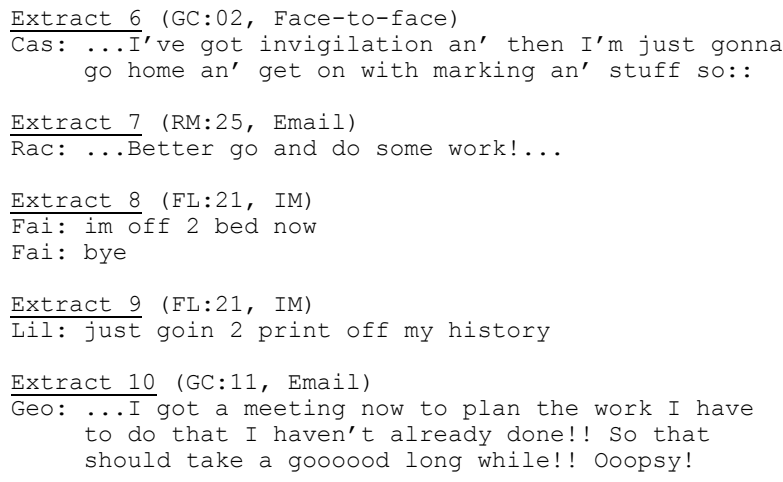

Current and future activities are often included in a single communication. For example, Extracts 1 and 3 above are taken from a longer stretch of interaction:

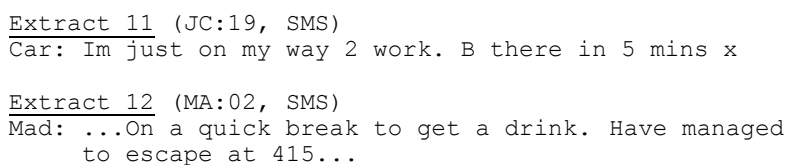

Although the content of these data fragments does not involve topicalising availability and capability to communicate, they provide recipients with information that allows them to infer when and how would be the best ways to contact these people. For example, in Extract 11, Carla's SMS informs Jess that she is currently contactable via the means of communication that are available to her during her journey to work. However, in five minutes Carla will be at work and the methods by which communication can be achieved will change. The exact nature of these changes (i.e., which methods of communication are available) are unspecified as her communication is designed for someone who knows her sufficiently well for this information not to need spelling out. This short SMS, then, provides a resource for Jess to surmise when and how to contact Carla.

In the cases above the information provided is a resource for recipients to make decisions about when and how would be the most appropriate way to communicate. However, this is a resource only when combined with the recipient's knowledge of the particular circumstances of the individual and in this way the originators recipient-design their communications.

\subsection{Unavailability and negotiating future communications}

As detailed above, people usually just describe activities and leave it to their recipients to infer information about their availability and capability to communicate. However, 
sometimes this implication is spelled out. Extracts 13-15 make clear the link between their activities and the consequential impact on their availability and capability to communicate.

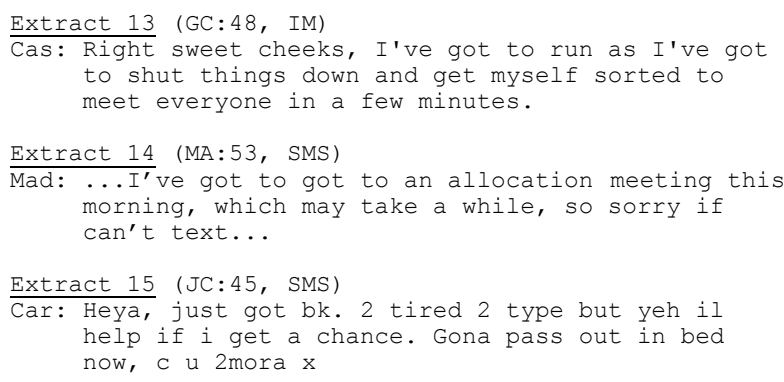

Extract 15 hints at the proposed next communication between the sender and the recipient (i.e. "c u 2mora") but in many instances this was more explicit. In addition to providing information about their own future activities, participants often combined this information with details of when and/or how they would contact their recipient next (Extracts 16-18) or request their recipient to make next contact (Extracts 19-20).

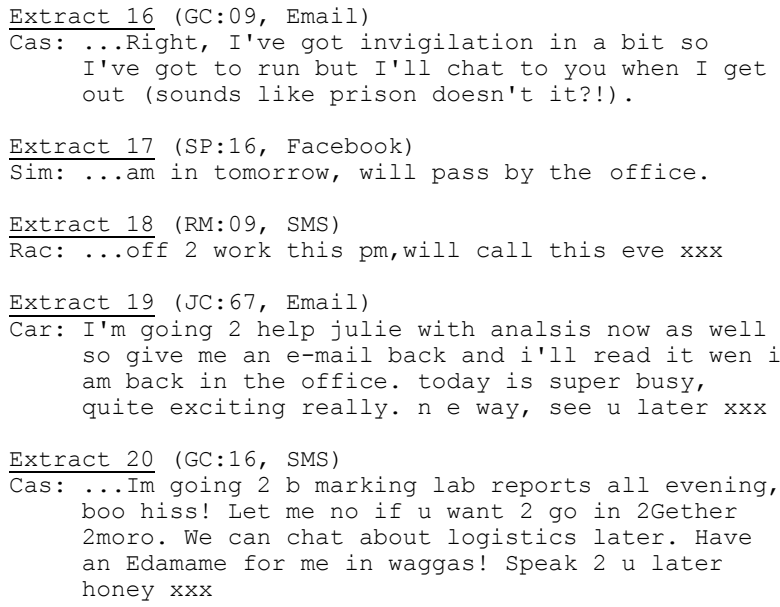

People's activities are not static and messages about plans and next contact are subject to negotiation. The data above are asynchronous but in synchronous interactions it is easier for participants to negotiate future contact taking into consideration each party's upcoming activities as Extract 21 illustrates:

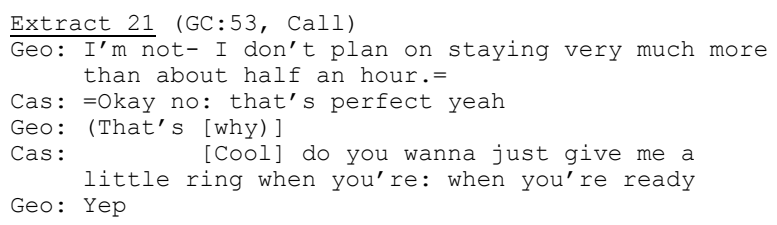

These interactions are negotiations that show participants' orientations to the relevance and significance of future availability and capability to communicate.

\subsection{Separating availability and capability}

Even when an interaction has been established successfully, participants may continue to ascertain availability:

Extract 22 (NC:07, call)

Cec: ...Are you having your dinner?...

Extract 22 shows the distinction between availability and capability to communicate. By answering, Nicky has made apparent that she is capable of communicating, but Cecily does not assume Nicky is available to communicate which is evidenced by Cecily's enquiry offering a candidate account for unavailability (i.e. 'having your dinner').

In sum, participants' communications about their current and future activities provides a rich resource for those with prior knowledge of them to make inferences about their availability and capability to communicate. Availability and capability are distinct elements - which are open to negotiation - that are integral to communication choices and, although they are generally not talked about in these terms, these concepts are relevant to assessing someone's presence (as Extract 22 shows).

\section{HOW PARTICIPANTS MAKE USE OF PRESENCE SYSTEMS}

Few mobile phones give availability information, but there is a default expectation that mobile phones are 'always on' [9]. So, unless a person is otherwise engaged in an activity that precludes mobile phone use then it is expected that contact can be made this way. It is expected that a person will always be capable of mobile phone contact. IM applications normally give presence information and have some automated features but they may be overridden by the user. However, the presence information that is given on many instant messaging systems is often not regarded as very useful [1]. Social networking sites usually give information as to whether a person is online.

The instant messaging systems used by participants all showed whether their contacts were online. However, participants did not always treat those listed as 'offline' as actually offline.

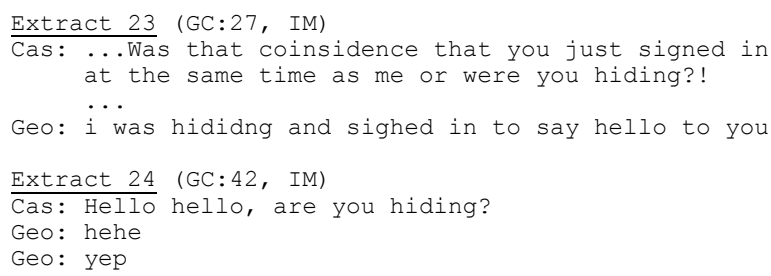

This lack of trust in the validity of the 'offline' listing means that participants may pursue an instant messaging session with an absent intended interlocutor which is of course unsuccessful (as happened in the following extract, for example).

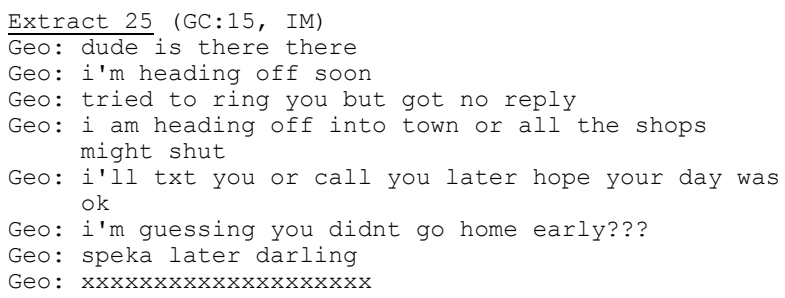

It is not only the online/offline distinction that is treated as inaccurate; participants also queried 'busy' statuses.

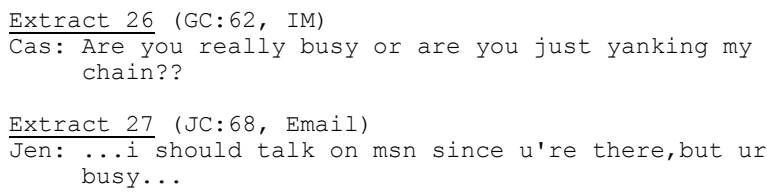

As well as treating others' displayed availability as inaccurate, they also overwrote their own. In an email to Cecily, Nicky accounts for the fact that she is listed as offline despite the evidence of the email showing that she is online with reference to the fact that she should actually be doing homework.

Extract 28 (NC:15, Email)

Nic: ...I am supposed to be doing my homework now but 
heyho this is more fun (just in case you're wondering why I'm not online)...

Status information varies depending on the recipient: there is no single description for everyone. This email shows that despite people's routine questioning of the displayed availability status there is still the underlying assumption that people do check what is listed. This is evidenced by Nicky's assumption that Cecily will have already been aware of her 'offline' status.

\section{CONCLUSIONS}

Although "[1]earning a person's availability is a very hard task" [5], it is one that humans achieve adeptly. The real difficulty lies in the creation of a system that incorporates this level of information.

Perhaps most significantly, the work presented here highlights the importance of communicating information that allows future availability and capability to be discerned. People plan their activities and this provides a resource for others who want to plan their communications and make decisions about media choice. Since it is important for a presence system to be easy to read [6] [10], an ideal system would indicate current and future information in a way that requires little interpretation to read (interpretation of the implications of that information is acceptable as this is what people are used to).

Previous research suggests a "sender calls when their situation is conducive to communication, but they do so with little knowledge of the receiver's situation" [5]. However, this was not the case for the participants in this data set. In interviews, it was almost universally reported that each participant had a rough understanding of the other's daily routines and, when this is coupled with the interactional data which shows regular updating about people's activities, we can see that they do have knowledge of their recipient's likely situation. This may be one of the ways in which social communications differ from workplace interactions in which the extent of personal knowledge may be less. A system cannot replace the complex interpretations and prior knowledge that exists in periphery of human consciousness but it should be a conduit or support for that information.

These findings support the requirement of a good presence system that "allow[s] users to manage expressions of presence to different groups and across different communication channels" [3]. We can see already that people do manipulate presence systems to manage their availability and capability to communicate profiles depending on the individual who is accessing that information. Therefore, an ideal system would allow for group and individual differentiation. Although since control and privacy are important requirements [11], the ability to override the automatic functions is important. In this way, the system needs to suggest rather than direct. These presence indicators are largely confined to methods of communication facilitated by the internet. However, these data show that people's communication that index availability and capability to communicate are trans-media. Therefore, an ideal presence system would require interaction across all media types.

\section{ACKNOWLEDGEMENTS}

This work is supported by British Telecommunications plc in a Knowledge Transfer Partnership with Digital World Research Centre (ref: KTP1346). We are grateful to the participants who volunteered to record their communications for this project.

\section{REFERENCES}

[1] Aswath, R. Presence vs. availability (http://blog.enthinnai.com/2007/10/21/ presence-vsavailability/) (October 21, 2007).

[2] Bentley, F. and Metcalf, C. J. Location and activity sharing in everyday mobile communication. In Proceedings of ACM CHI 2008 on Human Factors in Computing Systems (Florence, Italy, April 5-10, 2008). ACM Press, New York, 2008, 2453-2462.

[3] Chung, Y., Zimmerman, J., and Forlizzi, J. Monitoring and managing presence in incoming and outgoing communication. In Proceedings of ACM CHI 2005 on Human Factors in Computing Systems (Portland, Oregon, April 2-7, 2005). ACM Press, New York, 2005, 12841287.

[4] Dabbish, L., and Kraut, R. Coordinating communication: awareness displays and interruption. In Proceedings of ACM CHI 2003 on Human Factors in Computing Systems (Ft. Lauderdale, Florida, April 5-10, 2003). ACM Press, New York, 2003, 786-787.

[5] Danninger, M., Kluge, T., and Stiefelhagen, R. MyConnector: analysis of context cues to predict human availability for communication. In Proceedings of International Conference on Multimodal Interfaces (Banff, Canada, November 2-4, 2006): 12-19.

[6] Dey, A. K., and De Guzman, E. S. From awareness to connectedness: the design and deployment of presence displays. In Proceedings of ACM CHI 2006 on Human Factors in Computing Systems (Montreal, Canada, April 22-27, 2006). ACM Press, New York, 2006, 899-909.

[7] Dourish, P., and Bellotti, V. Awareness and coordination in shared workspaces. In Proceedings of ACM Conference on Computer-Supported Cooperative Work (Toronto, Canada, October 31 - November 4, 1992). ACM Press, New York, 1992, 107-114.

[8] Dourish, P. and Bly, S. Portholes: supporting awareness in a distributed work group. In Proceedings of $A C M \mathrm{CHI}$ 1992 on Human Factors in Computing Systems (Monterey, California, May 3-7, 1992). ACM Press, New York, 1992, 541-547.

[9] Fortunati, L. The mobile phone: an identity on the move. Personal and Ubiquitous Computing, 5, 2 (Jan 2001), 8598.

[10] Liechti, O. Awareness and the WWW: an overview. ACM SIGGROUP Bulletin, 21, 3, (Dec. 2000), 3-12.

[11] Milewski, A. E. and Smith, T. M. Providing presence cues to telephone users. In Proceedings of ACM Conference on Computer-Supported Cooperative Work (Philadelphia, Pennsylvania, December 2-6, 2000). ACM Press, New York, 2000, 89-96.

[12] Morse, D., Armstrong, S., and Dey, A. K. The what, who, where, when, why and how of context-awareness. In Proceedings of ACM CHI 2000 on Human Factors in Computing Systems (The Hague, The Netherlands, April 1-6, 2000). ACM Press, New York, 2000, 371.

[13] Oulasvirta, A., Raento, M., and Tiitta, S. ContextContacts: re-designing SmartPhone's contact book to support mobile awareness and collaboration. In Proceeding of MobileHCI '05 (Salzburg, Austria, September 22-29, 2005) ACM Press, New York, 2005, 167-174. 